\title{
A Note on Historical Terminology of the First Nuclear Age, I939-45
}

\section{ATOMIC URANIUM BOMB}

This is an explosive chain reaction taking place in pure or almost pure U235. It is produced by fast neutrons fissioning in a very short time the separated rare isotope U235, which is usually fissioned by slow neutrons of low energy. Heisenberg grasped this notion of a fast-neutron $\mathrm{U}_{235}$ bomb, but failed to understand that the critical mass required for such a bomb would be comparatively small.

\section{REACTOR}

During the first nuclear age $\left(1939^{-} 45\right)$ the reactor (or pile) was envisaged as a device to produce a chain reaction in a suitably arranged mass of unseparated uranium or uranium with an enriched $U_{235}$ content. The reaction was comparatively slow and nonexplosive, and understood to be generated by slow neutrons fissioning the $\mathrm{U}_{235}$ that was present. In order to prevent the fast neutrons produced by fission from losing the amount of energy that would result in their being captured uselessly in the predominant mass of $\mathrm{U}_{23} 8$ before reaching the lower energy where they would fission the $\mathrm{U}_{235}$, a moderator such as heavy water or graphite would be employed to absorb enough of the neutrons' energy to carry them below the $\mathrm{U}_{23} 8$ "resonance bands." This also improved the efficiency of the reaction.

\section{REACTOR-BOMB}

Before the scientific principle of the atomic uranium bomb was clearly understood (i.e., that fast neutrons produced in a small critical mass of U 235 an explosive chain reaction), suggestions were made as to how an explosion could be created in $\mathrm{U}_{235}$ by slow neutrons in a reactor stocked with either unseparated 
uranium or enriched uranium. In Germany research was pursued on the hypothesis that such a reactor-bomb would essentially be an unstable reactor using a moderator and highly enriched U235. This was seen in Germany as a solution to the insurmountable problem of obtaining the tons of $\mathrm{U}_{235}$ thought to be required for a $U_{235}$ bomb. 